\title{
Bioinspired self-repairing slippery surfaces with pressure-stable omniphobicity
}

\section{Citation}

Wong, Tak-Sing, Sung Hoon Kang, Sindy K. Y. Tang, Elizabeth J. Smythe, Benjamin D. Hatton, Alison Grinthal, and Joanna Aizenberg. 2011. Bioinspired Self-Repairing Slippery Surfaces with Pressure-Stable Omniphobicity. Nature 477, no. 7365: 443-447. doi:10.1038/nature10447.

\section{Published Version}

doi:10.1038/nature10447

\section{Permanent link}

http://nrs.harvard.edu/urn-3:HUL.InstRepos:27417441

\section{Terms of Use}

This article was downloaded from Harvard University's DASH repository, and is made available under the terms and conditions applicable to Open Access Policy Articles, as set forth at http:// nrs.harvard.edu/urn-3:HUL.InstRepos:dash.current.terms-of-use\#OAP

\section{Share Your Story}

The Harvard community has made this article openly available.

Please share how this access benefits you. Submit a story.

Accessibility 


\section{Bioinspired Self-Repairing Slippery Surfaces with Pressure-Stable Omniphobicity}

Tak-Sing Wong ${ }^{1}$, Sung Hoon Kang ${ }^{1}$, Sindy K. Y. Tang ${ }^{1}$, Elizabeth J. Smythe ${ }^{2}$, Benjamin D. Hatton ${ }^{1}$, Alison Grinthal ${ }^{1}$, and Joanna Aizenberg ${ }^{{ }^{*}}$

${ }^{1}$ School of Engineering and Applied Sciences and Wyss Institute for Biologically Inspired Engineering, Harvard University, Cambridge, Massachusetts 02138, USA

${ }^{2}$ Schlumberger-Doll Research Center, Schlumberger, Cambridge, Massachusetts 02139, USA

*To whom correspondence should be addressed. Email: jaiz@seas.harvard.edu

Word Count (including Abstract): 2157

Word Count (excluding Abstract): 1861

Word Count in Captions: 664

Number of Figures: 4 
Creating a robust synthetic surface that repels various liquids would have broad technological implications for areas ranging from biomedical devices to fuel transport to architecture but has proven to be extremely challenging. ${ }^{1}$ Inspirations from natural nonwetting structures, ${ }^{2-6}$ particularly the lotus, surged the development of liquid-repellent microtextured surfaces that rely on the formation of a stable air-liquid interface. ${ }^{7-9}$ Despite over a decade of intense research, these surfaces are, however, still plagued with problems that restrict their practical applications: they show limited oleophobicity with high contact angle hysteresis; ${ }^{9}$ fail under pressure ${ }^{10-12}$ and upon any physical damage; ${ }^{1,7,11}$ cannot self-heal, and are expensive to produce. ${ }^{1,11}$ To address these challenges, here we report a strategy to create self-healing, slippery liquid-infused porous surfaces (SLIPS) with exceptional liquid- and ice-repellency, pressure stability, and enhanced optical transparency. Our approach-inspired by Nepenthes pitcher plants ${ }^{13}$-is conceptually different from the lotus effect in that we use nano/microstructured substrates infused with a lubricating fluid. We define the materials requirements for which the lubricant is locked in place by the substrate and forms a stable, defect-free, inert "slippery" interface. This surface outperforms its natural counterparts $^{2-6}$ and state-of-the-art synthetic surfaces ${ }^{8,9,14-16}$ in its capability to repel various simple and complex liquids (water, hydrocarbons, crude oil, and blood); maintain low contact angle hysteresis $\left(<2.5^{0}\right)$; restore liquid-repellency after physical damage rapidly (within 0.1-1 s); resist ice adhesion; and function at high pressures (up to $\sim 676 \mathrm{~atm}$ ). We show that these properties are insensitive to the precise geometry of the underlying substrate, making our approach applicable to various inexpensive, low-surface-energy structured materials (e.g. porous Teflon membrane) that can be turned into robust omniphobic surfaces. We envision that the slippery surfaces can find important applications in fluid handling and transportation, optical sensing, medicine, and as selfcleaning and anti-fouling surfaces operating in extreme environments. 
Cutting-edge development of synthetic liquid-repellent surfaces is inspired by the lotus effect: ${ }^{2}$ water droplets are supported by surface textures on a composite solid/air interface that enables them to roll off easily. ${ }^{17,18}$ However, this approach, while promising, suffers from inherent limitations that severely restrict its applicability. First, trapped air is a largely ineffective cushion against organic liquids or complex mixtures that, unlike water, have low surface tension that strongly destabilizes suspended droplets. ${ }^{19}$ Moreover, the air trapped within the texture cannot stand up against pressure, so that liquids, particularly those with low surface tension, can easily penetrate the texture under even slightly elevated pressures or upon impact, ${ }^{10}$ conditions commonly encountered with driving rain or in underground transport pipes. Furthermore, synthetic textured solids are prone to irreversible defects arising from mechanical damage and fabrication imperfections; ${ }^{1,11}$ since each defect enhances the likelihood of the droplet pinning and sticking in place, textured surfaces are not only difficult to optimize for liquid mobility but inevitably stop working over time as irreparable damages accumulate. Recent progress in pushing these limits with increasingly complex structures and chemistries remains outweighed by substantial tradeoffs in physical stability, optical properties, large-scale feasibility, and/or difficulty and expense of fabrication. $8,9,14,15$

Nature, however, offers a remarkably simple alternative idea that has nothing to do with the lotus effect yet again capitalizes on microtextures: instead of using the structures to repel impinging liquids directly, systems such as the Nepenthes pitcher plant use them to lock-in an intermediary liquid that then acts by itself as the repellent surface. ${ }^{13}$ Well-matched solid and liquid surface energies, combined with the microtextural roughness, create a highly stable state in which the liquid fills the spaces within the texture and forms a continuous overlying film. ${ }^{20}$ In pitcher plants, this film is aqueous and effective enough to cause insects that step on it to slide from the rim into the digestive juices at the bottom by repelling the oils on their feet. ${ }^{21}$ 
Inspired by this idea, we report a synthetic liquid-repellent surface-which we name Slippery Liquid-Infused Porous Surface (SLIPS) — that consists of a film of lubricating liquid locked in place by a micro/nanoporous substrate (Fig. 1a). The premise for our design is that a liquid surface is intrinsically smooth and defect-free down to the molecular scale; provides immediate self-repair by wicking into damaged sites in the underlying substrate; is largely incompressible; and can be chosen to repel immiscible liquids of virtually any surface tension. We show that SLIPS create a smooth, stable interface that nearly eliminates pinning of the liquid contact line for both high- and low-surface-tension liquids, minimizes pressure-induced impalement into the porous structures, self-heals and retains its function following mechanical damage, and can be made optically transparent.

We designed the SLIPS based on three criteria: 1) the lubricating liquid must imbibe into, wet, and stably adhere within the substrate, 2) the solid must be preferentially wetted by the lubricating liquid rather than by the liquid one wants to repel, and 3) the lubricating and impinging test liquids must be immiscible. The first requirement is satisfied by using micro/nanotextured, rough substrates whose large surface area, combined with chemical affinity for the liquid, facilitates complete wetting by, and adhesion of, the lubricating fluid (Fig. S1). ${ }^{22,23}$ To satisfy the second criterion-the formation of a stable lubricating film that is not displaced by the test liquid (Fig. 1b)—we determine the chemical and physical properties required for working combinations of substrates and lubricants. We compare the total interfacial energies between textured solids that are completely wetted by either an arbitrary immiscible liquid $\left(E_{\mathrm{A}}\right)$, or a lubricating fluid with $\left(E_{1}\right)$ or without $\left(E_{2}\right)$ a fully wetted immiscible test liquid floating on top of it. To ensure the solid is wetted preferentially by the lubricating fluid one should have $\Delta E_{1}=E_{\mathrm{A}}-E_{1}>0$ and $\Delta E_{2}=E_{\mathrm{A}}-E_{2}>0$. The equations can be expressed as (see Supplementary Discussion): ${ }^{24}$

$$
\begin{aligned}
& \Delta E_{1}=R\left(\gamma_{\mathrm{B}} \cos \theta_{\mathrm{B}}-\gamma_{\mathrm{A}} \cos \theta_{\mathrm{A}}\right)-\gamma_{\mathrm{AB}}>0, \\
& \Delta E_{2}=R\left(\gamma_{\mathrm{B}} \cos \theta_{\mathrm{B}}-\gamma_{\mathrm{A}} \cos \theta_{\mathrm{A}}\right)+\gamma_{\mathrm{A}}-\gamma_{\mathrm{B}}>0,
\end{aligned}
$$


where $\gamma_{\mathrm{A}}$ and $\gamma_{\mathrm{B}}$ are the surface tensions for the test liquid to be repelled and the lubricating fluid, $\gamma_{\mathrm{AB}}$ is the interfacial tension at the liquid-liquid interface, $\theta_{\mathrm{A}}$ and $\theta_{\mathrm{B}}$ are the equilibrium contact angles of the immiscible test liquid and the lubricating fluid on a flat solid surface, and $R$ is the roughness factor, i.e., the ratio between the actual and projected surface areas of the textured solids. ${ }^{22}$

Based on these principles, we fabricated a set of SLIPS designed to repel liquids spanning a broad range of surface tensions. To generate roughness, we tested two types of porous solids, periodically ordered and random: (i) arrays of nanoposts functionalized with a low-surface-energy polyfluoroalkyl silane, ${ }^{25}$ and (ii) a random network of Teflon nanofibres distributed throughout the bulk substrate (Fig. 1c). For the lubricating film, we chose low-surface-tension perfluorinated liquids (e.g. $3 \mathrm{M}^{\mathrm{TM}}$ Fluorinert ${ }^{\mathrm{TM}}$ FC-70, $\gamma_{\mathrm{B}}=17.1 \mathrm{mN} / \mathrm{m}$; or Dupont ${ }^{\mathrm{TM}} \operatorname{Krytox}^{\circledR}$ oils) that are non-volatile and are immiscible with both aqueous and hydrocarbon phases and therefore able to form a stable, slippery interface with our solid substrates (i.e., $\Delta E_{1}>0$ and $\Delta E_{2}>0$ ) for a variety of polar and non-polar liquids including water, acids and bases, alkanes, alcohols, and ketones (Fig. 1d, 2a, b). The SLIPS were generated through liquid imbibition into the porous materials, ${ }^{23}$ resulting in a homogeneous and nearly molecularly smooth surface with a roughness of $\sim 1 \mathrm{~nm}$ (Fig. S2).

Each of these SLIPSs exhibits extreme liquid repellency as signified by very low contact angle hysteresis (CAH, $\Delta \theta<2.5^{\circ}$, Fig. 2a, b) and by very low sliding angles $\left(\alpha \leqslant 5^{\circ}\right.$ for droplet volume $\geqslant 2$ $\mu \mathrm{L}$, Fig. S3) against liquids of surface tension ranging from $\sim 17.2 \pm 0.5 \mathrm{mN} / \mathrm{m}$ (i.e., n-pentane) to 72.4 $\pm 0.1 \mathrm{mN} / \mathrm{m}$ (i.e., water). $\mathrm{CAH}$, the difference between the advancing and receding contact angles of a moving droplet, and sliding angle, the surface tilt required for droplet motion, directly characterize resistance to mobility; ${ }^{26}$ the low values therefore confirm a lack of pinning, consistent with a nearly defect-free surface. ${ }^{27}$ Based on the measured $\mathrm{CAH}$ and droplet volume $(\sim 4.5 \mu \mathrm{L})$, the estimated liquid retention force ${ }^{28}$ on SLIPS is $0.83 \pm 0.22 \mu \mathrm{N}, n=6$. This performance is nearly an order of magnitude 
better than the state-of-the-art lotus-leaf-inspired omniphobic surfaces, whose liquid retention forces are of the order of $5 \mu \mathrm{N}$ for low-surface-tension liquids (i.e., $\gamma_{\mathrm{A}}<25 \mathrm{mN} / \mathrm{m}$ ) at similar liquid volumes. ${ }^{9}$ Moreover, the liquid-repellency of SLIPS is insensitive to texture geometry (Fig. 2b), provided that the lubricating layer covers the textures (Fig. S4). This further confirms that liquid repellency is primarily conferred by the lubricating film, with the porous solid playing a secondary, but critically important role of immobilizing the film. Additionally, unlike lotus-based omniphobic surfaces where CAH depends on liquid surface tension and increases dramatically upon decrease of surface tension (Fig. 2b), such a dependence is absent for SLIPSs due to the chemical homogeneity and physical smoothness of the liquidliquid interface.

Experiments performed in a pressurized nitrogen environment show that SLIPS are capable of repelling water and liquid hydrocarbons both at and while transitioning to a pressure of $\sim 676$ atm (the highest available pressure in our setup). This is equivalent to the hydrostatic pressure at a depth of $\sim 7 \mathrm{~km}$ (Fig. 2c, Movie S1). To our knowledge, the highest recorded pressure stability of a superhydrophobic surface for water is $\sim 7 \mathrm{~atm}^{16}$ However, it is important to note that pressure stability for structured surfaces decreases drastically for liquids with low surface tension. For example, recent pressure stability studies of omniphobic surfaces based on impacting hexadecane droplets and evaporating octane droplets demonstrated stability up to only 400 to $1400 \mathrm{~Pa}$ (i.e., $4 \times 10^{-3}$ to $1.4 \times 10^{-2} \mathrm{~atm}$ ). ${ }^{9,10}$ While the reported omniphobic surfaces fail upon dynamic impact of low-tension liquids, ${ }^{10}$ SLIPS effectively repel impacting droplets for a wide assortment of liquid hydrocarbons (Fig. S5).

The lubricating film also serves as a self-healing coating to rapidly restore the liquid-repellent function following damage of the porous material by abrasion or impact. Due to the fluidic nature of the lubricating layer, the liquid simply flows toward the damaged area by surface energy-driven capillary action, ${ }^{29}$ and spontaneously refills the physical voids. As observed by high-speed camera imaging, the 
measured self-recovery time for a $\sim 50 \mu \mathrm{m}$ fluid displacement of the FC-70 lubricating layer on an epoxyresin-based SLIPS is $\sim 150$ ms (Fig. 3a). ${ }^{15}$ Even more impressively, SLIPS can repeatedly restore their liquid-repellent function upon recurring, large-area physical damage (Fig. 3b, Fig. S6, Movie S2).

We further demonstrate that, by choosing substrate and lubricant materials with matching refractive indices, SLIPS can be engineered for enhanced optical transparency in visible and/or nearinfrared wavelengths (Fig. 3c-e). Optical transparency is challenging to achieve through superhydrophobic surfaces, as they require nanostructures with dimensions under the sub-diffraction limit $(<\sim 100 \mathrm{~nm})^{30}$; the large difference in refractive index at the solid/air interface of these structured surfaces results in significant light scattering that reduces light transmission (Fig. 3c-e).

In addition to repelling liquids in their pure forms, SLIPS effectively repel complex fluids, such as crude oil (Fig. 4a, Movie S3) and blood (Fig. 4b, Movie S4), that rapidly wet and stain most existing surfaces. SLIPS also repel ice (Fig. 4c, Movie S5) and can serve as anti-sticking, slippery surfaces for insects (Fig. 4d, Movie S6) - a direct mimicry of pitcher plants. The omniphobic nature of our SLIPS also helps to protect the surface from a wide range of particulate contaminants by allowing self-cleaning by a broad assortment of fluids that collect and remove the particles from the surface (Fig. S7, Movie S7). Any of these capabilities could be compromised over time if the lubricant evaporates or is lost due to shearing under high flow conditions; choosing a lubricant with a minimal evaporation rate or an enhanced viscosity, or integrating the SLIPS with a fluid reservoir that enables continual self-replenishing (Fig. S8), enables prolonged operation.

No synthetic surface reported to date possesses all the unique characteristics of SLIPS: negligible contact angle hysteresis for low-surface-tension liquids and their complex mixtures, low sliding angles, instantaneous and repeatable self-healing, extreme pressure stability, and optical transparency. Our bioinspired SLIPS, which are prepared simply by infiltrating low-surface-energy porous solids with 
lubricating liquids, provide a straightforward and versatile solution for liquid repellency and resistance to fouling. Since low-surface-energy porous solids are abundant and commercially available, and the structural details are irrelevant to the resulting performance, one can turn any of these solids into highly omniphobic surfaces without the need to access expensive fabrication facilities. Since any liquid film is inherently smooth, self-healing, and pressure resistant, the lubricant can be chosen to be either biocompatible, index-matched with the substrate, optimized for extreme temperatures, or otherwise suitable for specific applications. With a broad variety of commercially available lubricants that possess a range of physical and chemical properties, we are currently exploring the limits of SLIPS's performance for long-term operation and under extreme conditions, such as high flow, turbulence, and high or low temperature environments. It is anticipated that SLIPS can be developed to serve as omniphobic materials capable of meeting emerging needs in biomedical fluid handling, fuel transport, anti-fouling, anti-icing, self-cleaning windows and optical devices, and many more areas that are beyond the reach of current technologies. 


\section{METHODS SUMMARY}

The lubricating fluids used for the experiments were perfluorinated fluids (e.g., $3 \mathrm{M}^{\mathrm{TM}}$ Fluorinert ${ }^{\mathrm{TM}}$ FC-70, Dupont ${ }^{\mathrm{TM}} \mathrm{Krytox}^{\circledR} 100$ and 103). Two types of porous solids were used in the experiments, periodically ordered epoxy-based nanostructured surfaces and random network of Teflon nanofibrous membranes. Specifically, Teflon membranes with average pore size of $\geqslant 200 \mathrm{~nm}$ and thickness of $\sim 60-80 \mu \mathrm{m}$ were purchased from Sterlitech Corporation, WA, USA. These membranes were used as received without further modification (SLIPS 1 sample). For the epoxy-based nanostructured surfaces, they were made from silicon masters through replica molding method ${ }^{25}$. The resulting dimensions of the nanostructures in epoxy replica were diameter of $\sim 300 \mathrm{~nm}$, height of $5 \mu \mathrm{m}$, and pitch of $2 \mu \mathrm{m}$ for the SLIPS 2 sample, and diameter of $\sim 300 \mathrm{~nm}$, height of $\sim 500 \mathrm{~nm}$ to $2 \mu \mathrm{m}$, and pitch of $\sim 900 \mathrm{~nm}$ for the SLIPS 3 sample, respectively. The epoxy replicas were further rendered hydrophobic by putting the samples in a vacuum desiccator overnight with a glass vial having $0.2 \mathrm{~mL}$ heptadecafluoro-1,1,2,2tetrahydrodecyltrichlorosilane (available from Gelest, Inc.). To prepare SLIPS, lubricating fluid was added onto the porous solids to form an over-coated layer. With matching surface chemistry and roughness, the fluid will spread spontaneously onto the whole substrate through capillary wicking. The thickness of the over-coated layer can be controlled by the fluid volume given a known surface area of the sample. Further details of the methods are available in Supplementary Information. 


\section{REFERENCES}

1 Quere, D. Wetting and roughness. Annu. Rev. Mater. Res. 38, 71-99 (2008).

2 Barthlott, W. \& Neinhuis, C. Purity of the sacred lotus, or escape from contamination in biological surfaces. Planta 202, 1-8 (1997).

3 Gao, X. F. \& Jiang, L. Water-repellent legs of water striders. Nature 432, 36-36 (2004).

4 Hansen, W. R. \& Autumn, K. Evidence for self-cleaning in gecko setae. Proc. Natl. Acad. Sci. USA 102, 385-389 (2005).

5 Gao, X. F., Yan, X., Yao, X., Xu, L., Zhang, K., Zhang, J. H., Yang, B. \& Jiang, L. The dry-style antifogging properties of mosquito compound eyes and artificial analogues prepared by soft lithography. Adv. Mater. 19, 2213-2217 (2007).

6 Epstein, A. K., Pokroy, B., Seminara, A. \& Aizenberg, J. Bacterial biofilm shows persistent resistance to liquid wetting and gas penetration. Proc. Natl. Acad. Sci. USA 108, 995-1000 (2011).

7 Quere, D. Non-sticking drops. Rep. Prog. Phys. 68, 2495-2532 (2005).

8 Tuteja, A., Choi, W., Ma, M. L., Mabry, J. M., Mazzella, S. A., Rutledge, G. C., McKinley, G. H. \& Cohen, R. E. Designing superoleophobic surfaces. Science 318, 1618-1622 (2007).

9 Tuteja, A., Choi, W., Mabry, J. M., McKinley, G. H. \& Cohen, R. E. Robust omniphobic surfaces. Proc. Natl. Acad. Sci. USA 105, 18200-18205 (2008).

10 Nguyen, T. P. N., Brunet, P., Coffinier, Y. \& Boukherroub, R. Quantitative testing of robustness on superomniphobic surfaces by drop impact. Langmuir 26, 18369-18373 (2010).

11 Bocquet, L. \& Lauga, E. A smooth future? Nature Mater. 10, 334-337 (2011).

12 Poetes, R., Holtzmann, K., Franze, K. \& Steiner, U. Metastable Underwater Superhydrophobicity. Phys. Rev. Lett. 105, 166104 (2010).

13 Bohn, H. F. \& Federle, W. Insect aquaplaning: Nepenthes pitcher plants capture prey with the peristome, a fully wettable water-lubricated anisotropic surface. Proc. Natl. Acad. Sci. USA 101, 14138-14143 (2004).

14 Ahuja, A., Taylor, J. A., Lifton, V., Sidorenko, A. A., Salamon, T. R., Lobaton, E. J., Kolodner, P. \& Krupenkin, T. N. Nanonails: A simple geometrical approach to electrically tunable superlyophobic surfaces. Langmuir 24, 9-14 (2008).

15 Li, Y., Li, L. \& Sun, J. G. Bioinspired self-healing superhydrophobic coatings. Angew. Chem. Int. Ed. 49, 6129-6133 (2010).

16 Lee, C. \& Kim, C. J. Underwater Restoration and Retention of Gases on Superhydrophobic Surfaces for Drag Reduction. Phys. Rev. Lett. 106, 014502 (2011).

17 Cassie, A. B. D. \& Baxter, S. Wettability of porous surfaces. Trans. Faraday Soc. 40, $0546-0550$ (1944).

18 Cassie, A. B. D. \& Baxter, S. Large contact angles of plant and animal surfaces. Nature 155, 2122 (1945).

19 Shafrin, E. G. \& Zisman, W. A. Constitutive relations in the wetting of low energy surfaces and the theory of the retraction method of preparing monolayers. J. Phys. Chem. 64, 519-524 (1960).

20 Bauer, U. \& Federle, W. The insect-trapping rim of Nepenthes pitchers: Surface structure and function. Plant Signal. Behav. 4, 1019-1023 (2009).

21 Federle, W., Riehle, M., Curtis, A. S. G. \& Full, R. J. An integrative study of insect adhesion: Mechanics and wet adhesion of pretarsal pads in ants. Integr. Comp. Biol. 42, 1100-1106 (2002). Wenzel, R. N. Resistance of solid surfaces to wetting by water. Ind. Eng. Chem. 28, $988-994$ (1936). 
23 Courbin, L., Denieul, E., Dressaire, E., Roper, M., Ajdari, A. \& Stone, H. A. Imbibition by polygonal spreading on microdecorated surfaces. Nature Mater. 6, 661-664 (2007).

24 de Gennes, P.-G., Brochard-Wyart, F. \& Quere, D. Capillarity and Wetting Phenomena : Drops, Bubbles, Pearls, Waves (Springer, 2003).

25 Pokroy, B., Epstein, A. K., Persson-Gulda, M. C. M. \& Aizenberg, J. Fabrication of Bioinspired Actuated Nanostructures with Arbitrary Geometry and Stiffness. Adv. Mater. 21, 463-469 (2009).

26 Chen, W., Fadeev, A. Y., Hsieh, M. C., Oner, D., Youngblood, J. \& McCarthy, T. J. Ultrahydrophobic and ultralyophobic surfaces: Some comments and examples. Langmuir 15, 3395-3399 (1999).

27 Delmas, M., Monthioux, M. \& Ondarcuhu, T. Contact Angle Hysteresis at the Nanometer Scale. Phys. Rev. Lett. 106, 136102 (2011).

28 Furmidge, C. G. Studies at phase Interfaces. 1. Sliding of liquid drops on solid surfaces and a theory for spray retention. J. Coll. Sci. 17, 309-324 (1962).

29 Ishino, C., Reyssat, M., Reyssat, E., Okumura, K. \& Quere, D. Wicking within forests of micropillars. Europhys. Lett. 79, 56005 (2007).

30 Nakajima, A., Fujishima, A., Hashimoto, K. \& Watanabe, T. Preparation of transparent superhydrophobic boehmite and silica films by sublimation of aluminum acetylacetonate. $A d v$. Mater. 11, 1365-1368 (1999). 
Supplementary Information is linked to the online version of the paper at www.nature.com/nature.

\section{Acknowledgements}

T.-S.W. acknowledges funding support from the Croucher Foundation Postdoctoral Fellowship. We thank Kristopher Eric Martin for the help with the drop impact test. We also thank James C. Weaver and Peter Allen for the help in manuscript preparation. We acknowledge the use of the facilities at the Harvard Center for Nanoscale Systems supported by National Science Foundation under the award number ECS-0335765. The work was partially supported by the Air Force Office of Scientific Research under the award number FA9550-09-1-0669-DOD35CAP (optical properties of SLIPS).

\section{Author Contributions}

T.-S.W. and J.A. perceived the concepts of the research. J.A. supervised the research. T.-S.W., S.H.K., S.K.Y.T. designed the experiments. T.-S.W. carried out surface wettability characterizations. S.H.K. prepared samples and conducted data analysis. T.-S.W., S.H.K., and S.K.Y.T. carried out surface morphology characterizations. T.-S.W. and S.H.K. carried out drop impact tests and ice experiments. E.J.S. and T.-S.W. carried out the high pressure and optical transmission measurements. B.D.H. and T.S.W. carried out blood compatibility tests. T.-S.W., S.H.K., A.G., and J.A. wrote the manuscript.

\section{Author Information}

Reprints and permissions information is available at www.nature.com/reprints. The authors declare no competing financial interests. Readers are welcome to comment on the online version of this article at www.nature.com/nature. Correspondence and requests for materials should be addressed to J.A. (jaiz@seas.harvard.edu). 


\section{FIGURE LEGENDS}

Figure 1. Design of SLIPS. a, Schematics showing the fabrication of a SLIPS by infiltrating a functionalized porous/textured solid with a low-surface-energy, chemically inert liquid to form a physically smooth and chemically homogeneous lubricating film on the surface of the substrate (see

Methods Summary). b, Schematics and time-lapse images showing the stability and displacement of lubricating films on silanized and non-silanized textured epoxy substrates, respectively. Dyed pentane was used to enhance visibility. c, Scanning electron micrographs showing the morphologies of porous/textured substrate materials: an epoxy resin-based nanofabricated post array (left) and a Teflonbased porous nanofiber network (right). d, Optical micrographs demonstrating the mobility of a lowsurface-tension liquid hydrocarbon, hexane $\left(\gamma_{\mathrm{A}}=18.6 \pm 0.5 \mathrm{mN} / \mathrm{m}\right.$, volume $\left.\approx 3.6 \mu \mathrm{L}\right)$, sliding on a SLIPS at a low angle $\left(\alpha=3.0^{\circ}\right)$.

Figure 2. Omniphobicity and high pressure stability of SLIPS. a, Time sequence images comparing mobility of pentane droplets $\left(\gamma_{\mathrm{A}}=17.2 \pm 0.5 \mathrm{mN} / \mathrm{m}\right.$, volume $\left.\approx 30 \mu \mathrm{L}\right)$ on a SLIPS and a superhydrophobic, air-containing Teflon porous surface. While pentane is repelled on the SLIPS, it wets and stains the traditional superhydrophobic surface. b, Comparison of contact angle hysteresis as a function of surface tension of test liquids (indicated) on SLIPS and on an omniphobic surface reported in Ref. 9. In the inset, advancing and receding contact angles of a liquid droplet are denoted as $\theta_{\text {adv }}$, and $\theta_{\text {rec, }}$ respectively. SLIPS 1, 2, and 3 refer to the surfaces made of 1) Teflon porous membrane, 2) array of epoxy posts of geometry 1 (pitch $=2 \mu \mathrm{m}$; height $=5 \mu \mathrm{m}$; and post diameter $=\sim 300 \mathrm{~nm}$ ), and 3) array of epoxy posts of geometry 2 (pitch $=\sim 900 \mathrm{~nm}$; height $=\sim 500 \mathrm{~nm}$ to $2 \mu \mathrm{m}$; and post diameter $=\sim 300 \mathrm{~nm}$ ), respectively. c, A plot showing the high pressure stability of SLIPS, as evident from the low sliding angle of a decane droplet $\left(\gamma_{\mathrm{A}}=23.6 \pm 0.1 \mathrm{mN} / \mathrm{m}\right.$, volume $\left.\approx 3 \mu \mathrm{L}\right)$ subjected to pressurized nitrogen gas in a 
pressure chamber (Supplementary Methods, Movie S1). Error bars indicate standard deviations from at least seven independent measurements.

Figure 3. Self-healing and optical transparency of SLIPS. a, Time-lapse images showing self-healing capability of SLIPS from a $\sim 50 \mu \mathrm{m}$-wide physical damage on a time scale on the order of $100 \mathrm{~ms}$. b, Time-lapse images showing the restoration of liquid repellency of a SLIPS after physical damage, as compared to a typical hydrophobic flat surface on which oil remains pinned at the damage site (Movie S2). c, Optical images showing enhanced optical transparency of an epoxy-resin-based SLIPS (left) as compared to significant scattering in the non-infused superhydrophobic nanostructured surface (right) in the visible light range. d, Optical transmission measurements for epoxy-resin-based SLIPS in the visible light range (400-750 nm). e, Optical transmission measurements for a Teflon-based SLIPS in the nearinfrared range $(800-2300 \mathrm{~nm})$.

Figure 4. Repellency of complex fluids, ice, and insects by SLIPS. a, Movement of light crude oil on a substrate composed of SLIPS, superhydrophobic Teflon porous membrane (S.H.), and a flat hydrophobic surface. Note slow movement on and staining of the latter two regions (Movie S3). $\mathbf{b}$, Comparison of the ability to repel blood by SLIPS, superhydrophobic Teflon porous membrane, and a flat hydrophilic glass surface. Note slow movement on and staining of the latter two regions (Movie S4). c, Ice mobility on a SLIPS (highlighted in green) as compared to strong adhesion to epoxy-resin-based nanostructured superhydrophobic surface (highlighted in yellow, see also Movie S5). The experiments were performed outdoor (note snow on the background) when temperature and relative humidity were $-4{ }^{\circ} \mathrm{C}$ and $\sim 45 \%$, respectively. Note also the reduced frosting and the resulting transparency of the SLIPS. d, Demonstration of the inability of a carpenter ant to hold on to a SLIPS. The ant (and a drop of fruit jam it is attracted to) 
slide along the SLIPS when the surface is tilted (Movie S6). Note that the ant can stably attach to regular flat hydrophobic surfaces, such as Teflon. All scale bars represent $10 \mathrm{~mm}$. 

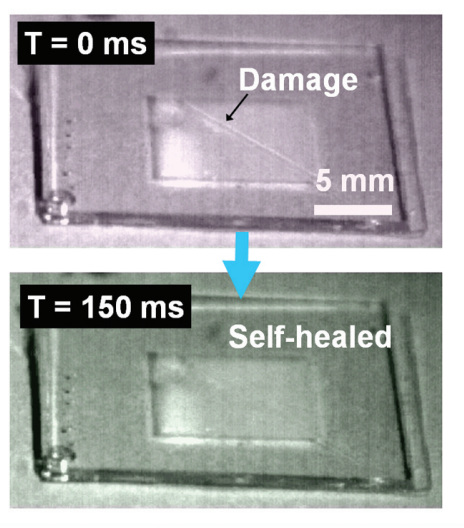

C

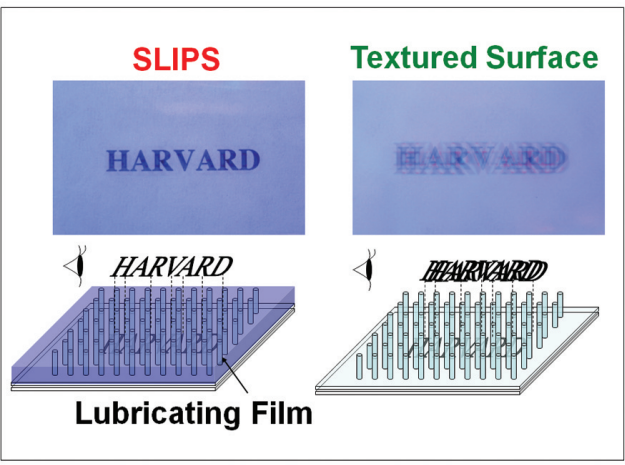

\section{SLIPS}
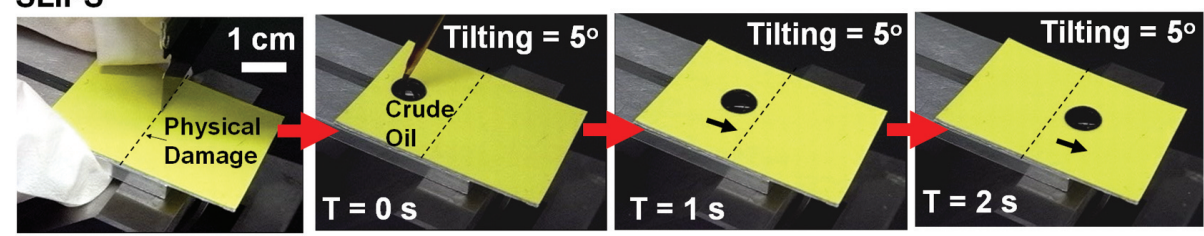

Teflon AF treated Flat Surface
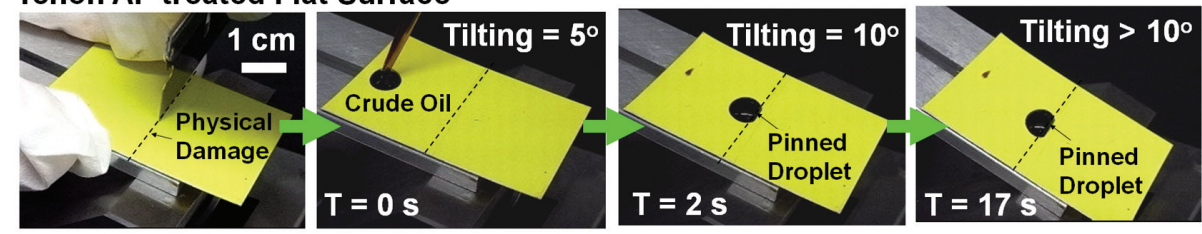

\section{d}
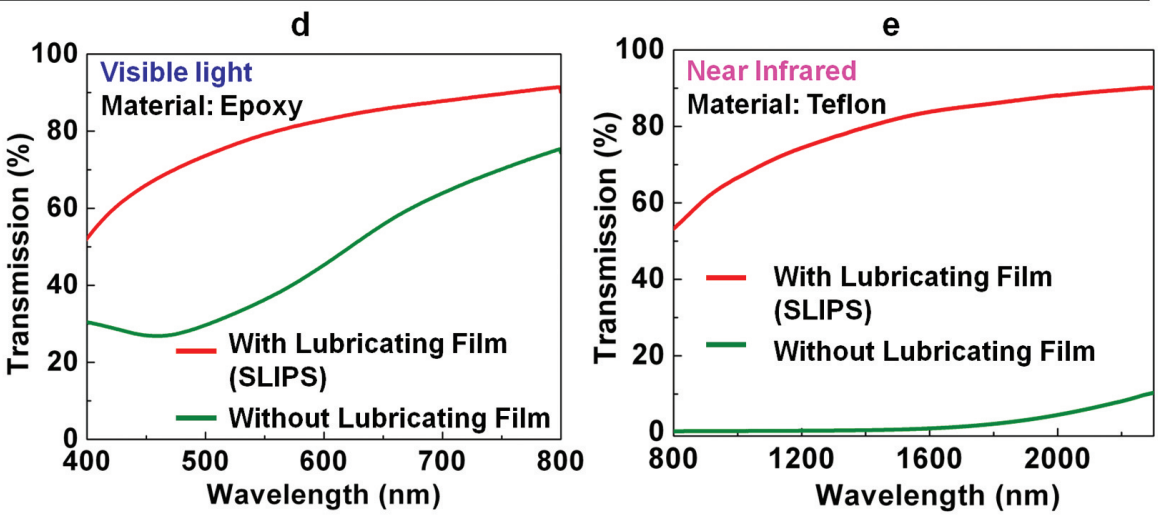
a

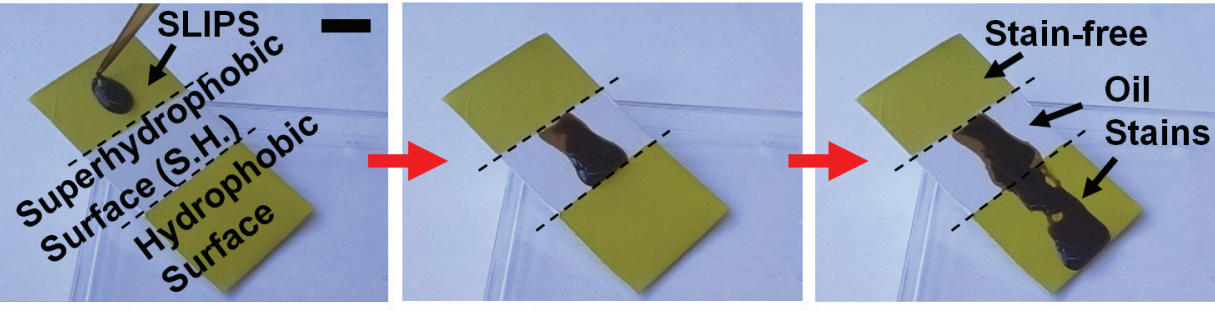

b

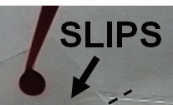

C
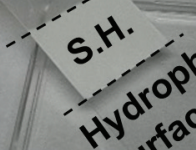

Lung
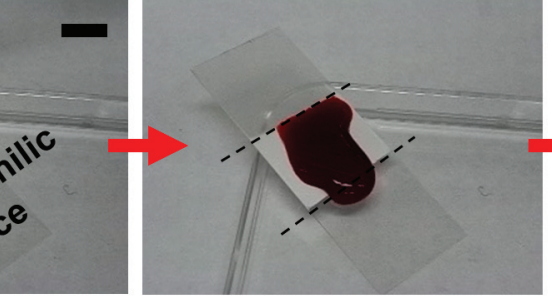

Stain-free

S.H.

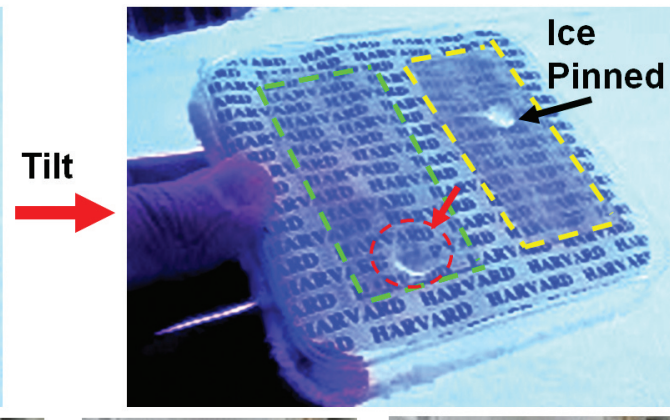

SLIPS

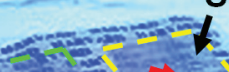

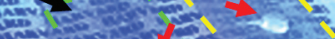

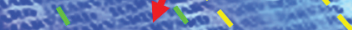

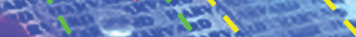

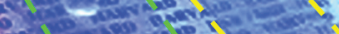

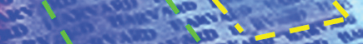

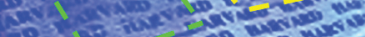

ist

(5) tis we..

ind and

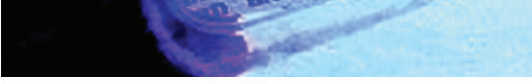

क.

SLIPS Ant
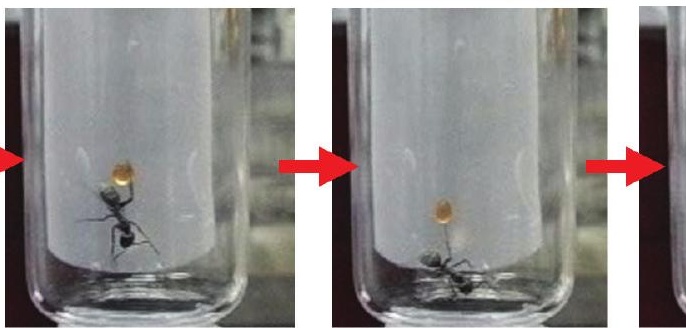